\title{
Three-dimensional atomic force microscopy mapping at the solid-liquid interface with fast and flexible data acquisition
}

\author{
Hagen Söngen, ${ }^{1,2, a)}$ Martin Nalbach, ${ }^{1}$ Holger Adam, ${ }^{1}$ and Angelika Kühnle ${ }^{1}$ \\ ${ }^{1}$ Institute of Physical Chemistry, Johannes Gutenberg University Mainz, Duesbergweg 10-14, \\ 55099 Mainz, Germany \\ ${ }^{2}$ Graduate School Materials Science in Mainz, Staudinger Weg 9, 55128 Mainz, Germany
}

(Received 5 April 2016; accepted 16 May 2016; published online 7 June 2016)

\begin{abstract}
We present the implementation of a three-dimensional mapping routine for probing solid-liquid interfaces using frequency modulation atomic force microscopy. Our implementation enables fast and flexible data acquisition of up to 20 channels simultaneously. The acquired data can be directly synchronized with commercial atomic force microscope controllers, making our routine easily extendable for related techniques that require additional data channels, e.g., Kelvin probe force microscopy. Moreover, the closest approach of the tip to the sample is limited by a user-defined threshold, providing the possibility to prevent potential damage to the tip. The performance of our setup is demonstrated by visualizing the hydration structure above the calcite (10.4) surface in water. Published by AIP Publishing. [http://dx.doi.org/10.1063/1.4952954]
\end{abstract}

\section{INTRODUCTION}

Dynamic atomic force microscopy (AFM) is a most valuable tool for studying solid-liquid interfaces in real space. Numerous technical improvements have made it possible to resolve mineral surfaces, ${ }^{1-3}$ molecular crystals, ${ }^{4}$ and molecular adsorbates ${ }^{5-8}$ with atomic-scale resolution. In addition, the introduction of three-dimensional (3D)-AFM by Fukuma et al. ${ }^{9}$ has enabled the visualization of the interfacial hydration structure. ${ }^{10-13}$

In $3 \mathrm{D}-\mathrm{AFM}$, the volume above the surface is scanned by the tip. This can be performed by collecting multiple images at different tip-sample distances. ${ }^{14,15}$ Since measurements in liquids are often subject to large drift, a more robust method is to periodically approach and retract the tip to and from the sample while laterally scanning across its surface. However, in contrast to conventional imaging, where a feedback loop is used to adjust the distance between tip and sample, a different method for regulating the tip-sample distance needs to be employed in 3D-AFM. One possibility is to use the distance feedback loop with low gain to adjust the average tipsample distance. This concept has been successfully applied in frequency modulation (FM)-AFM, ${ }^{9}$ bimodal $\mathrm{AFM},{ }^{16}$ and amplitude modulation AFM. ${ }^{13}$ Another option, which is more suited to non-planar samples, is to stop the cantilever approach whenever a user-defined threshold is reached. This opens up the possibility to investigate, e.g., step edges, other non-planar structures $^{17,18}$ and, additionally, allows to minimize potential damage to the tip. Kobayashi et al. ${ }^{19}$ employed a fieldprogrammable gate array in combination with a home-built AFM controller ${ }^{20}$ to perform threshold-based 3D mapping.

Here, similar to the above mentioned approach by Kobayashi et al., we present an easy-to-realize microcontroller-based implementation of 3D mapping at solidliquid interfaces. While being highly robust due to tip retrac-

a)Electronic mail: soengen@uni-mainz.de tion upon reaching a threshold, the main advantage of the present implementation is given by the large number of simultaneously recordable data channels that are directly synchronized with the scan movement. This provides means to employ more elaborate AFM-based measurement modes, e.g., related to the rapidly growing field of probing voltage-modulated electrostatic effects at solid-liquid interfaces. ${ }^{21-24}$

\section{IMPLEMENTATION}

The setup is based on a commercially available Multimode AFM with a Nanoscope V controller (Bruker Nano Surfaces Division, USA) optimized for low-noise operation in liquids using the FM operation mode..$^{25}$ This instrument has been further extended to perform photothermal cantilever excitation. ${ }^{26}$ However, the number of channels that can be recorded with the originally used controller is limited to four ( $z$-piezo displacement and three additional data channels), making the controller insufficient for some applications (e.g., 3D FMAFM). By combining custom electronics with an HF2LI lockin amplifier (Zurich Instruments, Switzerland), which also includes a phase-locked loop (PLL) and four feedback controllers, we are able to record an increased number of up to 20 channels. The setup is schematically depicted in Fig. 1 while its individual components are described in the following.

\section{A. FM-AFM operation}

For FM-AFM operation, the PLL of the HF2LI is used to excite the cantilever at its current eigen frequency. The amplitude of the oscillating cantilever is kept constant by adjusting the excitation amplitude using a feedback loop of the HF2LI. The eigen frequency shift $\Delta v_{\mathrm{e}}$ provided by the PLL is subtracted by an user-adjustable eigen frequency shift setpoint $\Delta v_{\mathrm{e}}^{\text {setpoint }}$. This signal is fed into the Nanoscope $\mathrm{V}$ controller using its external signal access module (SAM III, Bruker Nano Surfaces Division, USA). The controller nullifies the difference between eigen frequency shift and its setpoint by 


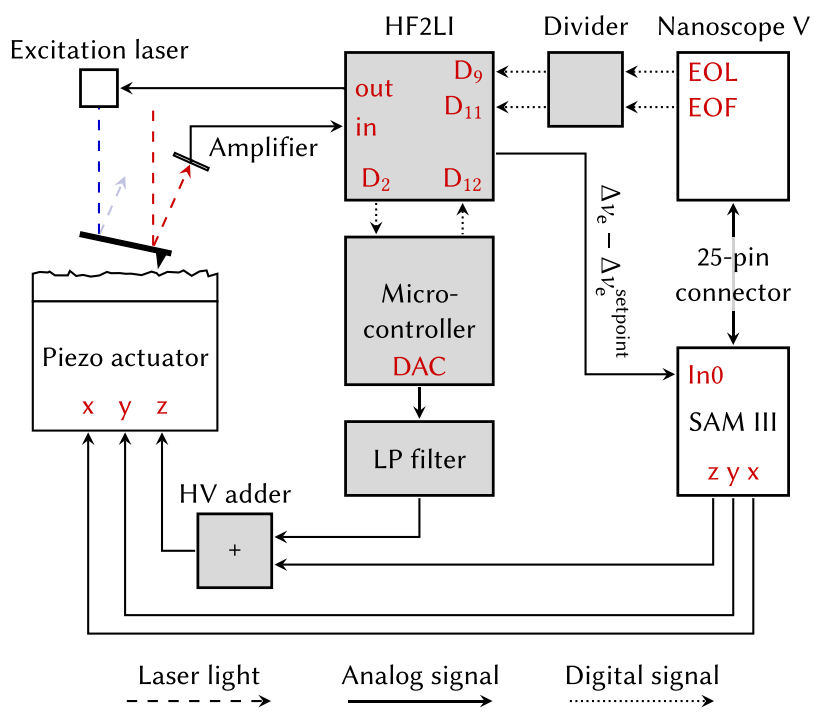

FIG. 1. Wiring diagram of the setup. The components added for 3D mapping are shaded gray. The laser used for photothermal excitation is described in Ref. 26, the amplifier of the deflection signal in Ref. 25. The Nanoscope V controller and the HF2LI are described in Sec. II, the signal access module (SAM III) in Sec. II A, the microcontroller and the low-pass (LP) filter in Sec. II B, and the divider in Sec. II C.

employing a feedback loop that adjusts the $z$-piezo displacement. While for conventional imaging, the tip is scanned at constant $\Delta v_{\mathrm{e}}$, we decrease the gain of the $z$-piezo feedback loop when performing 3D mapping. This ensures a compensation for sample tilt and vertical drift, but does not interfere with the mapping routine described below.

\section{B. Three-dimensional mapping in liquids}

We implement 3D mapping by modulating the z-piezo displacement with a microcontroller (Atmel SAM3X8E as provided with the Arduino Due platform) while the AFM controller ensures the lateral scanning of the tip over the surface. The modulation is performed by applying a voltage with a triangular waveform using the digital-to-analog (DAC) converter of the microcontroller. The triangular waveform ensures a uniform sampling of the entire range as well as a constant velocity for both the approaching and retracting tip. The vertical velocity, which can be set from a computer using a serial connection emulated over USB, is chosen to be larger (typically by a factor of 100) than the scan velocity in the fast scan direction. For noise reduction the bandwidth of the analog output of the microcontroller is limited to $10 \mathrm{kHz}$ by a secondorder low-pass (LP) filter based on the LT1363 operational amplifier (Linear Technology, USA). The filtered signal is added to the $z$-voltage provided by the AFM controller by using a high-voltage (HV) adder.

To prevent approaching the tip too close to the sample during the 3D mapping, a retraction criterion is provided by exploiting a feedback loop of the HF2LI. The feedback loop is adjusted as a pure proportional controller which considers the eigen frequency shift as its error value. If the eigen frequency shift exceeds a user-defined threshold, the feedback loop outputs a digital signal $\left(D_{2}\right.$ in Fig. 1$)$, which triggers an interrupt at the microcontroller. Subsequently, if the tip is approaching the sample when the interrupt is triggered, the direction of the vertical tip movement is reversed.

\section{Data acquisition and synchronization}

Data are acquired, processed, and displayed with a selfwritten software based on IGOR Pro (Wavemetrics, USA). A self-written IGOR Pro extension ${ }^{27}$ (written in C due to performance considerations) utilizes the HF2LI software interface to digitally capture data (i.e., eigen frequency shift, phase shift, oscillation amplitude, excitation amplitude, and static deflection) at a user-adjustable sampling rate of up to $28 \mathrm{kHz}$ per channel for up to 20 channels. The IGOR Pro extension runs as a separate thread in which the data are continuously polled from the HF2LI in an asynchronous fashion. Subsequently, the data are stored in cyclic buffers. To allow for an assignment of the recorded data with the position of the tip, a synchronization with both the scan engine of the AFM controller as well as the microcontroller that adjusts the $z$ piezo displacement is necessary. The synchronization with the scan engine is achieved by digital end-of-line (EOL) and endof-frame (EOF) triggers provided by the AFM controller. ${ }^{28}$ After each line, the AFM controller outputs a trigger pulse at its EOL output. Since the pulses are too short for a reliable detection, they are used as a clock signal for a binary counter (74HC4040, Fairchild Semiconductor, USA). The output $\mathrm{Q}_{0}$ of the counter (division by 2) represents each trace and retrace scan as a HIGH and LOW signal, respectively [ $x$-direction, Fig. 2(a)]. Thus, the fast lateral scan direction is directly available for further data assignment. Similarly, the AFM controller outputs two short digital LOW pulse after each frame. We use a second binary counter (which performs a division by 4 , output $\mathrm{Q}_{1}$ ) to obtain a HIGH and LOW signal for each up and

(a) line synchronization ( $x$-direction)

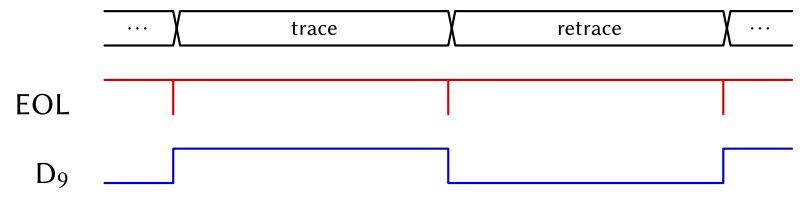

(b) frame synchronization ( $y$-direction)

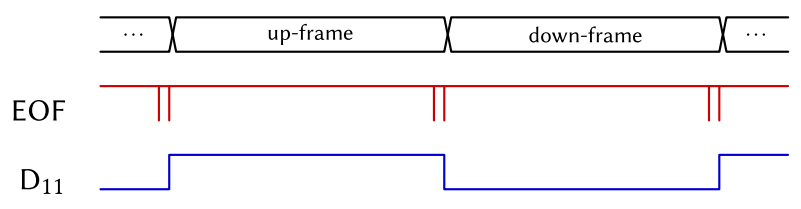

(c) vertical synchronization ( $z$-direction)

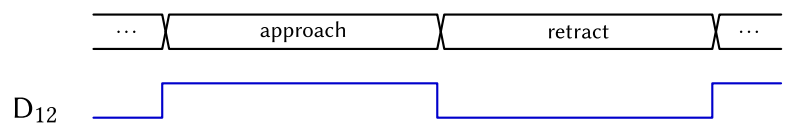

FIG. 2. Timing diagram of the line (a) and frame (b) synchronization of the scan controller as well as the vertical synchronization with the microcontroller (c). The naming of the digital inputs to the HF2LI $\left(D_{x}\right)$ is consistent with Fig. 1. 

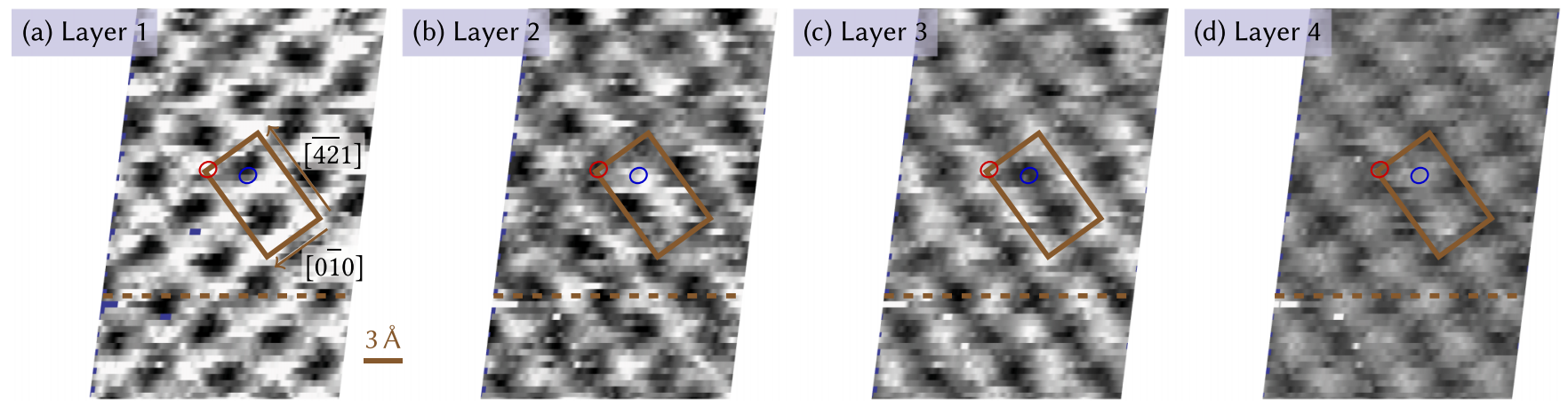

FIG. 3. Lateral slices extracted from a three-dimensional eigen frequency shift map above the calcite (10.4) surface in water (data are shown for the approaching tip). The black-to-white color scale is limited to values from (a) $-4.6 \mathrm{kHz}$ to $10.4 \mathrm{kHz}$, (b) $-4.6 \mathrm{kHz}$ to $6.4 \mathrm{kHz}$, (c) $-4.6 \mathrm{kHz}$ to $3.4 \mathrm{kHz}$, and (d) $-3.6 \mathrm{kHz}$ to $3.4 \mathrm{kHz}$.

down frame, respectively [y-direction, Fig. 2(b)]. Therefore, we directly obtain the slow lateral scan direction for further data assignment. The microcontroller indicates the direction of the vertical cantilever movement by an additional digital signal [z-direction, Fig. 2(c)]. The processed triggers from the EOL and EOF outputs of the AFM controller as well as the trigger of the microcontroller are fed into the digital inputs of the HF2LI. The HF2LI records the trigger channels as well as its other data channels which are used for further data processing within the self-written software.

Using the recorded triggers, we can assign the data to individual frames and lines. For each lateral scan line, the digitally encoded vertical direction [z-direction, Fig. 2(c)] is used to reconstruct the vertical position of the tip. We perform this line-by-line data processing online during the measurement, allowing the AFM user to quickly evaluate the data and react to potential changes during the recording of a 3D map.

\section{PROOF OF CONCEPT}

We demonstrate the 3D mapping capability of our setup by resolving the hydration structure of the calcite (10.4) surface in water. After cleaving the calcite crystal (Korth Kristalle $\mathrm{GmbH}$, Germany), pure water (Millipore GmbH, Germany) was injected in a closed liquid cell (Bruker Nano Surface Division, USA) that was sealed with an O-ring. The sample was allowed to equilibrate with the solution for approximately $6 \mathrm{~h}$. In water, the used silicon cantilever (PPP-NCHAuD, Nanosensors, Switzerland with a gold-coated backside) exhibited an eigen frequency of $129.6 \mathrm{kHz}$ and a quality factor of 9 . We determined the deflection sensitivity by a distancedependent deflection measurement while the tip was in contact with the surface and obtained a spring constant of $32.5 \mathrm{~N} \mathrm{~m}^{-1}$ by evaluating the thermal noise. ${ }^{29}$ The oscillation amplitude was kept constant at $0.11 \mathrm{~nm}$ and the eigen frequency shift retraction threshold was set to $20 \mathrm{kHz}$. An exemplary dataset is presented in Figs. 3 and 4.

The shown data were acquired in $160 \mathrm{~s}$ (corresponding to 64 scan lines and a time of $2.5 \mathrm{~s}$ per trace and retrace) with a vertical cantilever velocity of $92 \mathrm{~nm} \mathrm{~s}^{-1}$ (yielding approximately 190 pixel in vertical direction and 48 pixel in the fast scan direction). Due to the independent vertical and lateral tip movement, not the entire data are collected for the first and last approach or retract curve within each slice, respectively. This explains the additional regions with no data available (colorcoded blue) on the left and right edges in Figs. 3 and 4. To account for the static deflection, we use the sum of the adjusted $z$-piezo displacement $z_{\mathrm{p}}$ and the measured static deflection $q_{\mathrm{s}}$ as distance coordinate. Additionally, the data are corrected for sample tilt and vertical drift by subtracting the vertical distances by a linear fit of the position of closest approach to the sample. Moreover, through comparison with Ref. 30, the shown data were laterally distorted to match the surface unit cell dimensions of the calcite (10.4) surface. ${ }^{31}$

Lateral slices (extracted in various heights parallel to the sample surface) reveal a periodic pattern that allows to assign the surface unit cell of calcite [orange rectangle in Figs. 3(a)-3(d)]. The observed patterns are commensurate to each other. Moreover, at a fixed lateral position, minima and maxima alternate within the different layers. A vertical slice
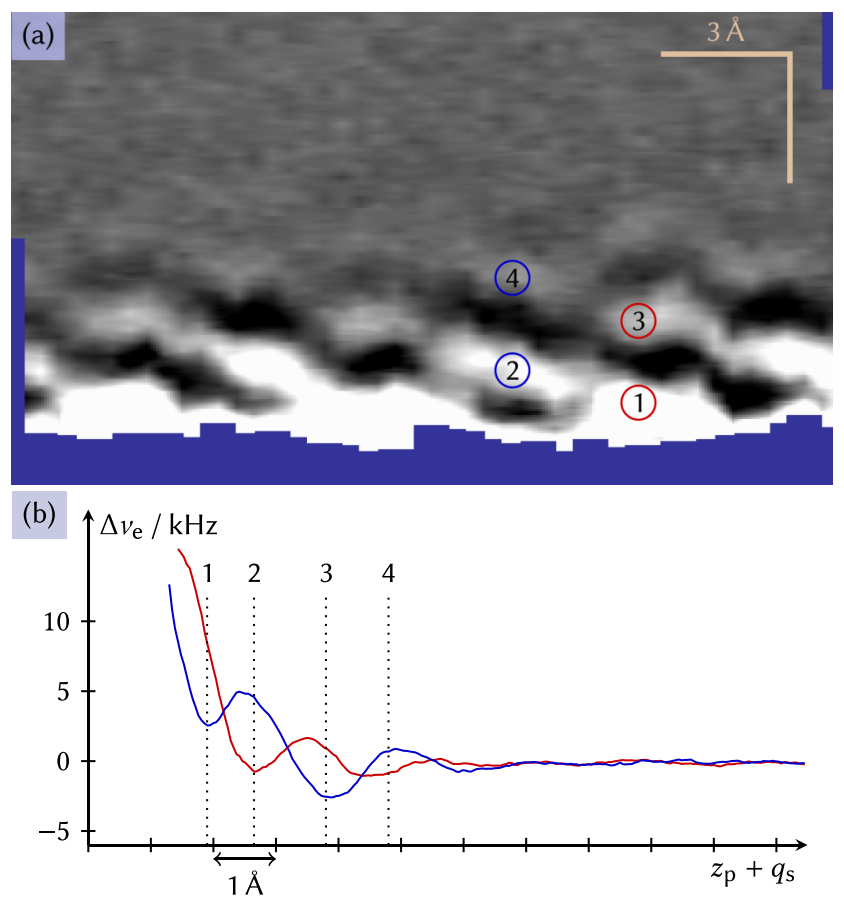

FIG. 4. (a) Vertical eigen frequency shift slice along the dashed line in Fig. 3. The black-to-white color scale is limited to values between $-3.6 \mathrm{kHz}$ and $5.4 \mathrm{kHz}$. Data points not accessed by the tip are colored blue. (b) Averaged profiles over the area indicated by the circles in Fig. 3. The indicated positions of the extrema in the profiles correspond to the heights of the lateral slices in Fig. 3. 
shown in Fig. 4(a) shows a regular checkerboard-like pattern of $\Delta v_{\mathrm{e}}$ extrema. In Fig. 4(b) we show the eigen frequency shift as a function of the tip-sample distance extracted above different positions within the surface unit cell (averaged within the blue and red circle shown in Fig. 3). Here, several alternating minima and maxima are again clearly visible. Thus, our observations are qualitatively in line with both previous experimental $^{12,13,32,30}$ and theoretical ${ }^{33,34,30} 3 \mathrm{D}$-AFM measurements on calcite (10.4), where water has been found to be vertically ordered in several layers as well as laterally commensurate to the underlying surface unit cell. Note that a direct quantitative comparison of the eigen frequency shift in FM-AFM would require also to compare several operational parameters (i.e., oscillation amplitude, excitation amplitude, and phase shift) which is beyond the scope of this manuscript.

\section{CONCLUSION}

In conclusion, we present the implementation of threedimensional mapping at solid-liquid interfaces using a custom data acquisition system. Up to 20 channels can be recorded simultaneously in synchronization with the scan movement. The $z$-piezo displacement is performed by a microcontroller which is able to reverse the vertical direction of the tip movement based on a user-defined threshold. This flexible architecture makes our setup easily extendable for techniques related to AFM, such as Kelvin probe force microscopy, open-loop electric potential measurements and bimodal AFM techniques. We demonstrate the high-resolution capabilities of our 3D FMAFM setup by successfully imaging the hydration structure of the calcite (10.4) surface in water.

\section{ACKNOWLEDGMENTS}

H.S. is a recipient of a fellowship through the Excellence Initiative (DFG/GSC 266). Financial support by the German Research Foundation through Grant No. KU1980/7-1 is gratefully acknowledged. We thank Stefanie Klassen for most helpful experimental support.

${ }^{1}$ T. Fukuma, K. Kobayashi, K. Matsushige, and H. Yamada, Appl. Phys. Lett. 87, 034101 (2005).

${ }^{2}$ S. Rode, N. Oyabu, K. Kobayashi, H. Yamada, and A. Kühnle, Langmuir 25, 2850 (2009).

${ }^{3}$ Y. Araki, K. Tsukamoto, N. Oyabu, K. Kobayashi, and H. Yamada, Jpn. J. Appl. Phys. 51, 08KB09 (2012).

${ }^{4}$ P. Spijker, T. Hiasa, T. Musso, R. Nishioka, H. Onishi, and A. S. Foster, J. Phys. Chem. C 118, 2058 (2014).

${ }^{5}$ T. Fukuma, A. S. Mostaert, L. C. Serpell, and S. P. Jarvis, Nanotechnology 19, 384010 (2008).
${ }^{6}$ M. Schreiber, M. Eckardt, S. Klassen, H. Adam, M. Nalbach, L. Greifenstein, F. Kling, M. Kittelmann, R. Bechstein, and A. Kühnle, Soft Matter 9 , 7145 (2013).

${ }^{7}$ M. Pfreundschuh, D. Alsteens, M. Hilbert, M. O. Steinmetz, and D. J. Müller, Nano Lett. 14, 2957 (2014).

${ }^{8}$ R. Momper, M. Nalbach, K. Lichtenstein, R. Bechstein, and A. Kühnle, Langmuir 31, 7283 (2015).

${ }^{9}$ T. Fukuma, Y. Ueda, S. Yoshioka, and H. Asakawa, Phys. Rev. Lett. 104, 016101 (2010).

${ }^{10}$ K. Kimura, S. Ido, N. Oyabu, K. Kobayashi, Y. Hirata, T. Imai, and H. Yamada, J. Chem. Phys. 132, 194705 (2010).

${ }^{11}$ H. Asakawa, S. Yoshioka, K. Nishimura, and T. Fukuma, ACS Nano 6, 9013 (2012).

${ }^{12}$ H. Imada, K. Kimura, and H. Onishi, Langmuir 29, 10744 (2013).

${ }^{13}$ C. Marutschke, D. Walters, J. Cleveland, I. Hermes, R. Bechstein, and A. Kühnle, Nanotechnology 25, 335703 (2014).

${ }^{14}$ B. J. Albers, T. C. Schwendemann, M. Z. Baykara, N. Pilet, M. Liebmann, E. I. Altman, and U. D. Schwarz, Nat. Nanotechnol. 4, 307 (2009).

${ }^{15}$ C. Moreno, O. Stetsovych, T. K. Shimizu, and O. Custance, Nano Lett. 15, 2257 (2015).

${ }^{16}$ E. T. Herruzo, H. Asakawa, T. Fukuma, and R. Garcia, Nanoscale 5, 2678 (2013).

${ }^{17}$ K. Umeda, K. Kobayashi, N. Oyabu, K. Matsushige, and H. Yamada, Nanotechnology 26, 285103 (2015).

${ }^{18}$ K. Suzuki, K. Kobayashi, N. Oyabu, K. Matsushige, and H. Yamada, J. Chem. Phys. 140, 054704 (2014)

${ }^{19}$ K. Kobayashi, N. Oyabu, K. Kimura, S. Ido, K. Suzuki, T. Imai, K. Tagami, M. Tsukada, and H. Yamada, J. Chem. Phys. 138, 184704 (2013).

${ }^{20}$ K. Kobayashi, H. Yamada, H. Itoh, T. Horiuchi, and K. Matsushige, Rev. Sci. Instrum. 72, 4383 (2001).

${ }^{21}$ N. Kobayashi, H. Asakawa, and T. Fukuma, Rev. Sci. Instrum. 81, 123705 (2010).

${ }^{22}$ A. L. Domanski, E. Sengupta, K. Bley, M. B. Untch, S. A. L. Weber, K. Landfester, C. K. Weiss, H.-J. Butt, and R. Berger, Langmuir 28, 13892 (2012).

${ }^{23}$ K. Umeda, K. Kobayashi, N. Oyabu, Y. Hirata, K. Matsushige, and H. Yamada, J. Appl. Phys. 116, 134307 (2014).

${ }^{24}$ L. Collins, S. Jesse, J. I. Kilpatrick, A. Tselev, O. Varenyk, M. B. Okatan, S. A. L. Weber, A. Kumar, N. Balke, S. V. Kalinin, and B. J. Rodriguez, Nat. Commun. 5, 3871 (2014).

${ }^{25}$ S. Rode, R. Stark, J. Lübbe, L. Tröger, J. Schütte, K. Umeda, K. Kobayashi, H. Yamada, and A. Kühnle, Rev. Sci. Instrum. 82, 073703 (2011).

${ }^{26}$ H. Adam, S. Rode, M. Schreiber, K. Kobayashi, H. Yamada, and A. Kühnle, Rev. Sci. Instrum. 85, 023703 (2014).

${ }^{27}$ The source code for the data acquisition system, the source code for the microcontroller, and additional configuration files are available on request from the first author.

${ }^{28}$ Most commercial AFM controllers provide similar signals that can be used for synchronization. Thereby, our implementation can easily be modified for other controllers.

${ }^{29}$ J. Lübbe, M. Temmen, P. Rahe, A. Kühnle, and M. Reichling, Beilstein J. Nanotechnol. 4, 227 (2013).

${ }^{30}$ T. Fukuma, B. Reischl, N. Kobayashi, P. Spijker, F. F. Canova, K. Miyazawa, and A. S. Foster, Phys. Rev. B 92 (2015).

${ }^{31}$ H. Effenberger, K. Mereiter, and J. Zemann, Z. Kristallogr. 156, 233 (1981).

${ }^{32}$ Y. Araki, K. Tsukamoto, R. Takagi, T. Miyashita, N. Oyabu, K. Kobayashi, and H. Yamada, Cryst. Growth Des. 14, 6254 (2014).

${ }^{33}$ T. D. Perry IV, R. T. Cygan, and R. Mitchell, Geochim. Cosmochim. Acta 71, 5876 (2007).

${ }^{34}$ S. Kerisit and S. C. Parker, Chem. Commun. 2004, 52. 